10 Milgrom H, Fowler-Taylor A, Vidaurre CF, et al. Safety and tolerability of omalizumab in children with allergic (IgE-mediated) asthma. Curr Med Res Opin 2011; 27: 163-169.

11 Chung KF, Wenzel SE, Brozek JL, et al. International ERS/ATS guidelines on definition, evaluation and treatment of severe asthma. Eur Respir J 2014; 43: 343-373.

12 Global Initiative for Asthma. Global Strategy for Asthma Management and Prevention. 2014. www.ginasthma.org

13 Lowe PJ, Renard D. Omalizumab decreases IgE production in patients with allergic (IgE-mediated) asthma; PKPD analysis of a biomarker, total IgE. Br J Clin Pharmacol 2011; 72: 306-320.

14 Molimard M, Mala L, Bourdeix I, et al. Observational study in severe asthmatic patients after discontinuation of omalizumab for good asthma control. Respir Med 2014; 108: 571-576.

15 Busse WW, Trzaskoma B, Omachi TA, et al. Evaluating Xolair persistency of response after long-term therapy (XPORT). Am J Respir Crit Care Med 2014; 189: A6576.

\title{
Bronchial thermoplasty in asthma: 2-year follow-up using optical coherence tomography
}

To the Editor:

Bronchial thermoplasty (BT) is a novel, nonpharmacological procedure for treatment of severe asthma. Recently, the Asthma Intervention Research 2 clinical trial demonstrated asthmatics had fewer hospitalisations following BT, which persisted 5 years after therapy [1]. However, it is well recognised that asthma is a heterogeneous disease with distinct asthma phenotypes and, not surprisingly, not all asthmatics in that trial benefited from BT [2].

Although bronchoscopic biopsies, pulmonary function tests, exhaled nitric oxide, sputum eosinophil counts and other biological measures have been proposed as biomarkers for evaluating treatment effects [3], these biomarkers cannot provide regional information to characterise airway remodelling in the targeted airways prior to and longitudinally following treatment. Although imaging approaches, such as computed tomography of the lung [4] and magnetic resonance imaging using inhaled contrast agents [5], do provide regional information, these tests are limited to indirect assessment of the small airways. Optical coherence tomography $(\mathrm{OCT})$ is a minimally invasive imaging technique for visualising airway wall structures with near-histological resolution [6-8]. OCT has been used for the evaluation of airway remodelling $[9,10]$ and early lung neoplastic changes $[8,11]$.

Identifying asthma phenotypes with the greatest response to BT is likely to bring the potential for better patient selection and ultimately better patient outcomes, and further research into methods capable of careful patient selection for BT has been strongly recommended [12]. Here, our objective was to provide a pilot study in two asthma patients who underwent BT in order to investigate the role of OCT imaging for evaluating airway remodelling prior to and longitudinally following BT treatment.

Two patients with chronic persistent asthma provided written informed consent. Flexible bronchoscopy was performed under local anaesthesia and conscious sedation [7]; BT was performed according to established protocols (Boston Scientific Corp., Marlborough, MA, USA) [2, 13]. OCT images of the subsegmental branch of the right-lower lobe (RB8a\&b and RB9a\&b) were acquired prior to and immediately after BT as well as at 3 weeks, 6 weeks, 6 months and 2 years post-BT using a custom-built swept-source OCT system [14] and a C7 Dragonfly Imaging Catheter (St Jude Medical Inc., St Paul, MN, USA). OCT airway segments matched by visual inspection at each time-point were selected for analysis. The lumen area $\left(A_{\mathrm{i}}\right)$ and outer wall area $\left(A_{\mathrm{o}}\right)$ for three consecutive OCT slices were manually segmented (ImageJ; National Institutes of Health, Bethesda, MD, USA) to generate airway wall (WA) percentage:

$$
\mathrm{WA}=\frac{A_{\mathrm{i}}}{A_{\mathrm{o}}} \times 100
$$

Patient A was a 51-year-old male with no smoking history and an asthma duration of 6 years. Patient B was a 56-year-old female and an ex-smoker at the time of the study (10 pack-years) with an asthma 
duration of 27 years. Both patients reported similar forced expiratory volume in $1 \mathrm{~s} \mathrm{(FEV1)} \mathrm{(patient} \mathrm{A:}$ $1.63 \mathrm{~L}$ ( $53 \%$ pred); patient B: $1.52 \mathrm{~L}$ (54\% pred)) as well as similar doses of inhaled steroids $(4000 \mu \mathrm{g}$ beclomethasone equivalent per day), salmeterol $(100 \mu \mathrm{g}$ per day) and tiotropium (18 $\mu \mathrm{g}$ per day). However, patient A reported higher doses of prednisone (patient A: $25 \mathrm{mg}$ per day; patient B: $5-10 \mathrm{mg}$ per day) and salbutamol (patient A: $3500-3700 \mu \mathrm{g}$ per day; patient B: $300-500 \mu \mathrm{g}$ per day) than patient B prior to BT.

Figure 1a shows OCT images pre-BT, immediately post-BT, and 3 weeks, 6 weeks, 6 months and 2 years post-BT for patients A and B. Patient A showed a remarkably thickened and inflamed epithelium, irregular basement membrane, and prominent smooth muscle pre-BT. Immediately post-BT, copious secretions and sloughing of the epithelium were observed. At 3 and 6 weeks post-BT, the epithelium appeared thinner and the lamina propria appeared thicker from deposition of collagen, and smooth muscle appeared slightly less prominent. At 6 months and 2 years post-BT, OCT showed recurrence of the inflammatory changes in the epithelium. In contrast to patient A, the epithelial layer of patient B was not inflamed pre-BT. Airway wall oedema was visually apparent 3 weeks post-BT but was reduced at week 6. The smooth muscle layer was also less prominent 6 weeks post-BT, and remained the same at 6 months and 2 years. At 6 months and 2 years post-BT, patient B also showed a normal bronchial epithelium with collagen deposition in the submucosa.

Figure $1 \mathrm{~b}$ shows OCT airway measurements pre-BT, immediately post-BT, and 3 weeks, 6 weeks, 6 months and 2 years post-BT for patients A and B. For Patient A, airway secretions prevented OCT airway measurements immediately post-BT. At week 3, OCT airway measurements showed an increase in WA. Although WA improved slightly at week 6, WA was increased 6 months and 2 years post-BT. In patient A, there was a transient improvement in FEV1 at 3 and 6 weeks post-BT (pre-BT: $1.63 \mathrm{~L} ; 3$ weeks post-BT: $2.14 \mathrm{~L}$; 6 weeks post-BT: $2.27 \mathrm{~L}$ ). However, symptoms recurred 4 months after treatment and patient A remained on $20-50 \mathrm{mg}$ per day of prednisone due to recurrent exacerbations. FEV1 was also worse 6 months and 2 years post-BT than pre-BT ( 6 months post-BT: $1.08 \mathrm{~L} ; 2$ years post-BT: $1.11 \mathrm{~L}$ ).

As shown in figure $1 \mathrm{~b}$ for patient $\mathrm{B}$, OCT WA was reduced at 3 and 6 weeks compared to baseline. This reduction in WA persisted 6 months and 2 years post-BT. FEV1 also progressively improved post-BT (pre-BT: $1.52 \mathrm{~L} ; 3$ weeks post-BT: $1.68 \mathrm{~L} ; 6$ weeks post-BT: $1.78 \mathrm{~L} ; 6$ months post-BT: $2.03 \mathrm{~L}$ ) but dropped slightly 2 years post-BT (2 years post-BT: $1.82 \mathrm{~L}$ ). Symptoms decreased along with discontinuation of prednisone and less frequent use of rescue medication.

Figure 1c shows histology of RB8 bronchial biopsies 6 months post-BT. For patient A, histology showed a partially denuded epithelium, thickened basement membrane, moderate-to-severe inflammation and remodelling of the submucosa by collagen deposition. In contrast, for patient $B$, histology of the same airway showed a normal bronchial epithelium with collagen deposition in the submucosa.

This pilot study illustrates, for the first time, that OCT can be used to evaluate in vivo airway remodelling longitudinally following BT in patients with severe asthma. Although the two asthmatics evaluated were similar in age and presented with similar clinical features and spirometry, the asthma patients demonstrated very different responses to BT as well as very distinct OCT airway wall features at baseline.

Following BT in patient A, despite evidence of FEV1 improvement at weeks 3 and 6, OCT imaging showed no reduction in airway wall thickness and the patient's symptoms returned 4 months post-treatment. In contrast, patient $\mathrm{B}$ showed progressive improvements in FEV1 for at least 6 months and reductions in OCT airway wall thickness, and demonstrated improvements in respiratory symptoms and decreased medication use for 2 years post-BT. Although patient A was a clear BT nonresponder, patient B demonstrated improvements following BT therapy that could not be predicted based on baseline spirometry or clinical features.

Although this study was limited to only two patients, it is certainly hypothesis generating and a number of questions arise from this pilot study. Most important is the question of whether OCT can identify characteristics within the airway wall that predict BT responders. Inflammation is visible on OCT $[7,15]$ and, notably, in this study, the BT responder showed thickened airway wall without evidence of inflammation in the epithelial layer at baseline compared to the nonresponder.

In summary, we evaluated two severe asthmatics immediately prior to and longitudinally following BT, and demonstrated a reduction in airway wall thickness that persisted 2 years following treatment in the BT responder, as well as differences in airway wall features between the responder and nonresponder prior to treatment. These observations generate hypotheses for a larger study to determine if airway changes defined by OCT imaging can identify asthma patients who will benefit from BT and to determine the long-term effects of the treatment. 

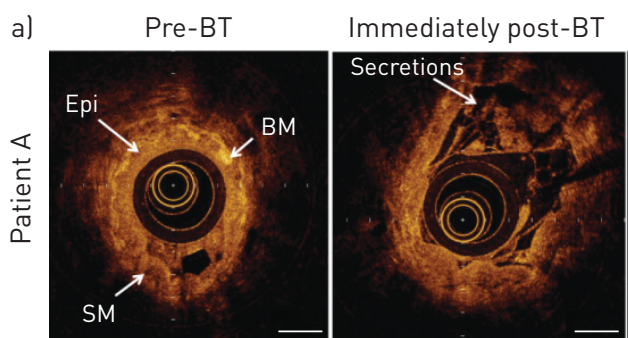

3 weeks post-BT
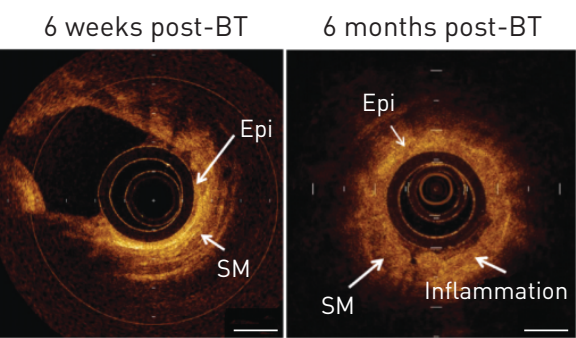

2 years post-BT

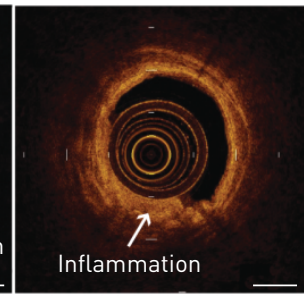

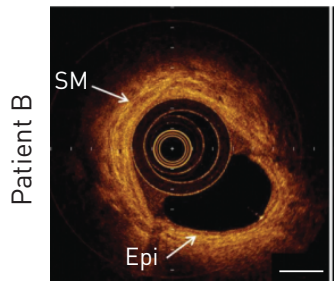
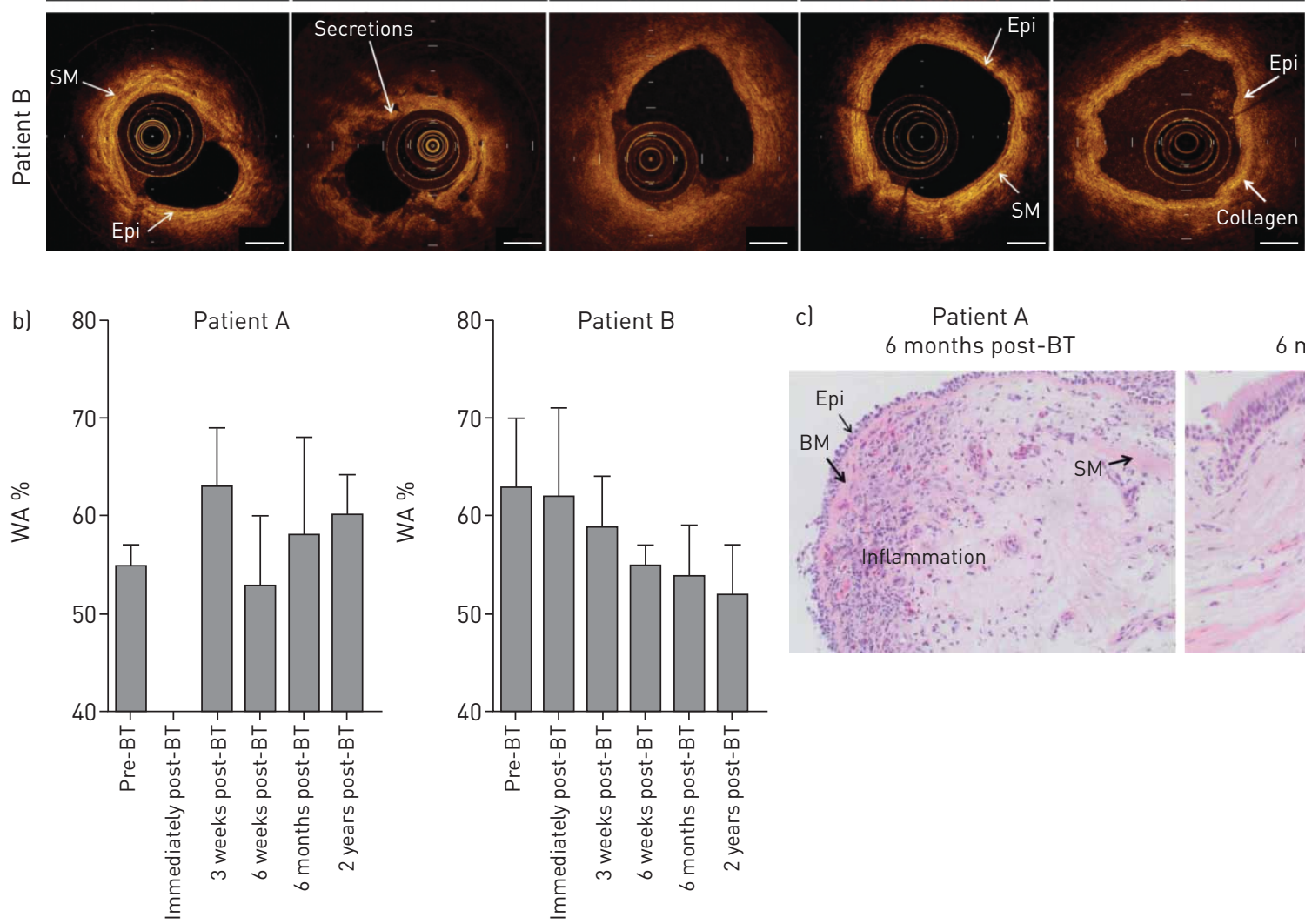

clo

Patient A 6 months post-BT

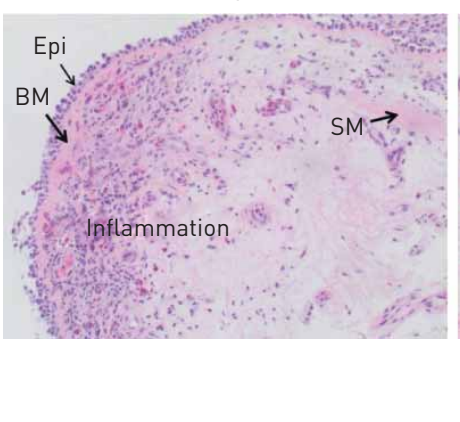

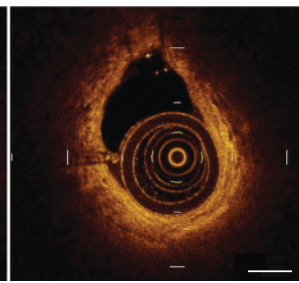

Patient B 6 months post-BT
FIGURE 1 a) Optical coherence tomography images (scale bars $=1 \mathrm{~mm}$ ) and b) mean \pm SD airway measurements prior to and immediately after bronchial thermoplasty (BT), 3 weeks post-BT, 6 weeks post-BT, 6 months post-BT and 2 years post-BT, with c) the corresponding bronchial biopsy at 6 months post-BT. Epi: epithelium; BM: basement membrane; SM: smooth muscle: WA: airway wall.
@ERSpublications

Optical coherence tomography small airway imaging may be used for better patient phenotyping and selection for BT http://ow.ly/O0Cm2

Miranda Kirby ${ }^{1}$, Keishi Ohtani ${ }^{2,3}$, Rosa Maria Lopez Lisbona ${ }^{3,4}$, Anthony M.D. Lee ${ }^{3}$, Wei Zhang ${ }^{3,5}$, Pierre Lane ${ }^{3}$, Nina Varfolomeva ${ }^{6}$, Linda $\mathrm{Hui}^{6}$, Diana Ionescu ${ }^{7}$, Harvey O. Coxson ${ }^{1}$, Calum MacAulay ${ }^{3}$, J. Mark FitzGerald ${ }^{6}$ and Stephen Lam $^{3,6}$

${ }^{1}$ Centre for Heart Lung Innovation, University of British Columbia and St. Paul's Hospital, Vancouver, BC, Canada. ${ }^{2}$ Department of Surgery, Tokyo Medical University, Tokyo, Japan. ${ }^{3}$ Imaging Unit, Integrative Oncology Department, British Columbia Cancer Research Centre, Vancouver, BC, Canada. ${ }^{4}$ Department of Respirology, Bellvitge University Hospital, Hospitalet de Llobregat, Barcelona, Spain. ${ }^{5}$ Department of Respiratory and Critical Care Medicine, Peking University First Hospital, Beijing, China. ${ }^{6}$ Institute for Heart and Lung Health, University of British Columbia and Vancouver General Hospital, Vancouver, BC, Canada. ${ }^{7}$ Department of Pathology, British Columbia Cancer Agency and the University of British Columbia, Vancouver, BC, Canada.

Correspondence: Stephen Lam, British Columbia Cancer Research Centre, 675 West 10th Avenue, Vancouver, BC, V5Z 1L3, Canada. E-mail: slam2@bccancer.bc.ca

Received: Jan 292015 | Accepted after revision: April 102015 | First published online: May 282014

Support statement: M. Kirby gratefully acknowledges postdoctoral support from the Canadian Institutes of Health Research (CIHR) Bisby award, the CIHR Integrated and Mentored Pulmonary and Cardiovascular Training program (IMPACT), and the Michael Smith Foundation for Health Research (MSFHR). We also gratefully acknowledge the 
CIHR-Thoracic Imaging Network of Canada and the United States Public Health Service contracts N01-CN 35000 from the National Cancer Institute for the OCT technology development. The funding sources for this study played no role in the design and conduct of the study; in the collection, management, analysis, and interpretation of the data; or in the preparation of the manuscript. The funding sources did not review the manuscript. S. Lam had full access to all of the data in the study and takes responsibility for the integrity of the data and the accuracy of the data analysis. Funding information for this article has been deposited with FundRef.

Conflict of interest: Disclosures can be found alongside the online version of this article at erj.ersjournals.com

Acknowledgements: We thank Myles McKinnon, Jennifer Campbell and Chulho Hyun (British Columbia Cancer Agency, Vancouver, BC, Canada) for their assistance with the bronchoscopy procedures.

\section{References}

1 Wechsler ME, Laviolette M, Rubin AS, et al. Bronchial thermoplasty: Long-term safety and effectiveness in patients with severe persistent asthma. J Allergy Clin Immunol 2013; 132: 1295-1302.

2 Castro M, Rubin AS, Laviolette M, et al. Effectiveness and safety of bronchial thermoplasty in the treatment of severe asthma: a multicenter, randomized, double-blind, sham-controlled clinical trial. Am J Respir Crit Care Med 2010; 181: 116-124.

3 Hanania NA, Wenzel S, Rosen K, et al. Exploring the effects of omalizumab in allergic asthma: an analysis of biomarkers in the EXTRA study. Am J Respir Crit Care Med 2013; 187: 804-811.

4 Nakano Y, Wong JC, de Jong PA, et al. The prediction of small airway dimensions using computed tomography. Am J Respir Crit Care Med 2005; 171: 142-146.

5 Kauczor HU, Ebert M, Kreitner KF, et al. Imaging of the lungs using 3He MRI: preliminary clinical experience in 18 patients with and without lung disease. J Magn Reson Imaging 1997; 7: 538-543.

6 Coxson HO, Quiney B, Sin DD, et al. Airway wall thickness assessed using computed tomography and optical coherence tomography. Am J Respir Crit Care Med 2008; 177: 1201-1206.

7 Lam S, Standish B, Baldwin C, et al. In vivo optical coherence tomography imaging of preinvasive bronchial lesions. Clin Cancer Res 2008; 14: 2006-2011.

8 Hariri LP, Applegate MB, Mino-Kenudson M, et al. Volumetric optical frequency domain imaging of pulmonary pathology with precise correlation to histopathology. Chest 2013; 143: 64-74.

9 Williamson JP, McLaughlin RA, Noffsinger WJ, et al. Elastic properties of the central airways in obstructive lung diseases measured using anatomical optical coherence tomography. Am J Respir Crit Care Med 2011; 183: 612-619.

10 Coxson HO, Lam S. Quantitative assessment of the airway wall using computed tomography and optical coherence tomography. Proc Am Thorac Soc 2009; 6: 439-443.

11 Tsuboi M, Hayashi A, Ikeda $\mathrm{N}$, et al. Optical coherence tomography in the diagnosis of bronchial lesions. Lung Cancer 2005; 49: 387-394.

12 British Thoracic Society, Scottish Intercollegiate Guidelines Network. British guideline on the management of asthma. Thorax 2014; 69: 1-192.

13 Cox G, Miller JD, McWilliams A, et al. Bronchial thermoplasty for asthma. Am J Respir Crit Care Med 2006; 173: 965-969.

14 Lee AM, Ohtani K, Macaulay $\mathrm{C}$, et al. In vivo lung microvasculature visualized in three dimensions using fiber-optic color Doppler optical coherence tomography. J Biomed Opt 2013; 18: 50501.

15 Whiteman SC, Yang Y, Gey van Pittius D, et al. Optical coherence tomography: real-time imaging of bronchial airways microstructure and detection of inflammatory/neoplastic morphologic changes. Clin Cancer Res 2006; 12: 813-818.

\section{Effective strategies for managing new Pseudomonas cultures in adults with cystic fibrosis}

To the Editor:

Pseudomonas aeruginosa is a common chronic pulmonary infection in cystic fibrosis (CF). It is associated with poor outcomes and new detection should prompt an early eradication attempt [1-3].

Strict segregation and eradication policies have led to a declining prevalence of chronic P. aeruginosa infection, with only $30 \%$ of CF adolescents having chronic infection when they transition to adult services [4]. Adult CF physicians increasingly manage new P. aeruginosa cultures in adults without robust evidence to guide decision-making. We evaluated the success of our eradication and suppression strategies for adults 